\title{
L'objet de la dépression
}

\author{
Alain Vanier
}

La notion contemporaine de dépression se développe à partir du milieu du $\mathrm{XX}^{\mathrm{e}}$ siècle et s'appuie sur la découverte des électrochocs, puis des antidépresseurs. Elle va progressivement s'autonomiser par rapport aux catégories nosographiques qui l'incluaient, tout d'abord par la mise en avant par les psychanalystes de la notion de pathologie narcissique, puis par la promotion d'une séméiologie débarrassée du problème étiologique, qui constitue le principe du DSM-III ${ }^{1}$. Ce mouvement s'accompagne d'un recul d'une conception du symptôme comme expression d'un conflit psychique que le traitement doit conduire à assumer comme sujet, au profit du retour de l'approche déficitaire qui avait prévalu tout au long du XIX ${ }^{\mathrm{e}}$ siècle. Devenu « objet de sa maladie », et non plus sujet confronté à des conflits psychiques dont il ne peut répondre, le patient déprimé « $a$ une pathologie dont on peut le débarrasser ${ }^{2} »$.

Mais, aujourd'hui, le succès de cette création nosologique a fait de la « dépression » un problème de santé publique. Campagnes de sensibilisation, plaquettes d'information se multiplient pour essayer de traiter cette sorte de paradoxe qui associe, en

1. A. Ehrenberg, La fatigue d'être soi. Dépression et société, Paris, Odile Jacob, 1998.

2. A. Ehrenberg, «Dépression », dans Dictionnaire de la pensée médicale, Paris, PUF, 2004. 
France, une consommation trop élevée de psychotropes, antidépresseurs surtout, et une augmentation des pathologies dites dépressives que cette surconsommation n'arrive pas à traiter. Il s'agirait de remédier à ce qu'un éminent psychiatre a appelé « dysconsommation » liée, comme en témoignent ces campagnes, à une méconnaissance de la dépression par les médecins qui prescriraient largement ces médicaments à des patients bien portants et omettraient de le faire aux patients déprimés véritables. Dans cette démarche, on n'interrogera pas la raison de cette surconsommation inappropriée d'antidépresseurs, puisque, plus ou moins implicitement, elle est attribuée à l'incompétence des médecins. On oubliera la remarque de Jean-Claude Ameisen rappelant ces études qui montrent que la production d'un nouveau médicament, comme les antidépresseurs, s'accompagne corrélativement d'une élévation considérable du seuil d'intolérance aux symptômes que ce nouveau médicament prétend traiter. On oubliera de se souvenir de la clinique ancienne qui montrait plutôt un continuum de l'humeur, et l'inscrivait comme un trait nécessairement associé à d'autres pour caractériser un tableau clinique. Aujourd'hui, il y a la dépression, accompagnée de spécifications grossières, telles que chacun peut s'y reconnaître. Serions-nous en train d'illustrer la formule de Swift : "Apollon, le dieu de la médecine, passait pour envoyer les maladies. À l'origine, les deux métiers n'en faisaient qu'un, et il en est toujours ainsi » ? Il arrive à chacun des jours plus sombres que d'autres, tribut de notre condition humaine. La dépression, l'humeur triste, devient une faute morale, non pas au sens que lui donnait Lacan, mais comme écart par rapport à une norme sociale médicalisée. Elle enjoint chacun « de se normaliser, c'est-à-dire de se conformer à une norme présentée comme scientifiquement définie ». Ainsi, « ordre moral et ordre médical échangent leurs valeurs », ajoute Dominique Lecourt ${ }^{3}$. En effet, « les temps modernes sont intolérants aux troubles psychiques ${ }^{4}$, note Paul Fréour, et la définition des maladies mentales se révèle essentiellement opérationnelle : entrent dans ce cadre ceux dont les troubles ne permettent pas

3. D. Lecourt, « Normes », dans Dictionnaire de la pensée médicale, op. cit.

4. P. Fréour, dans M. Sendrail (sous la direction de), Histoire culturelle de la maladie, Toulouse, Privat, 1980. 
l'adaptation à la vie sociale, scolaire ou professionnelle, familiale ou publique.»

Curieusement, on reste hésitant encore quant au but de ces campagnes. S'agit-il de rectifier le tir en traitant ceux qui seraient « vraiment malades », et non plus ceux qui souffrent seulement de la rigueur du monde contemporain, ou de résorber cette question par le traitement psychiatrique des maladies sociales d'aujourd'hui ? Aux dépressions pas véritablement caractérisées, qui ne relèveraient pas du traitement médicamenteux, on propose les psychothérapies dont la définition demeure floue, mais la fonction précise : un remède adaptatif au malaise social. Ainsi, la grande étude SMPG (Santé mentale en population générale), menée il y a cinq ans, à l'origine de ces campagnes, montrait que le risque dépressif s'accroît nettement chez les femmes, les personnes seules, ou au chômage. On peut supposer qu'il devient majeur si les trois caractères sont réunis. On n'est pas loin de Coluche. Comment ne pas évoquer le terme de biopolitique avancé par Foucault ? Pour lui, le monde moderne capitaliste n'a pas représenté « le passage d'une médecine collective à une médecine privée, mais l'inverse ». Il note, en effet, que le premier programme d'hygiène des populations apparaît au milieu du $\mathrm{XVIII}^{\mathrm{e}}$ siècle. Jusque-là, la monarchie n'avait pas comme premier souci de s'occuper de la santé des individus en tant que population, alors que l'État moderne devient de plus en plus responsable de notre «bien-être physique, mental et social » (OMS). C'est l'effet d'une corrélation entre une « individualisation toujours plus poussée » et « la consolidation de la communauté des individus comme totalité ». Lacan, prémonitoire, avait fait sienne la formule de Saint-Just : « Le bonheur est devenu un facteur de la politique. »

Avec ce souci de l'hygiène des populations et la promotion de normes collectives auxquelles il convient de s'adapter, le maître mot de la médecine moderne est la pédagogie, qui constitue aussi le modèle des nouvelles psychothérapies. C'est-à-dire obtenir un comportement standardisé de patients consentants. Le patient d'aujourd'hui est devenu un " consommateur de soins », « qui négocie sa prise en charge avec les soignants ${ }^{5} »$. A. Merlot cite

5. A. Merlot, « De l'observance », Nervure, ${ }^{\circ}$ 8, t.XX, novembre 2007. 
J. Palazzolo : «Le patient doit s'autogouverner de par son adhésion $^{6}$. » Mais s'autogouverner signifie-t-il autre chose qu'aller dans le sens voulu par la médecine ? C'est le mode du choix contemporain préempté par les experts : choisissez, mais il n'y a qu'une seule réponse valable 7 . "S'il n'est pas observant, il devient le seul fautif, consommateur incapable de se servir habilement de son pouvoir, mettant hors de cause le système de santé et les traitements eux-mêmes. » La pédagogie a pour but d'améliorer l'observance ; mais elle se fait « au détriment du travail relationnel entre le patient et ses soignants ${ }^{8} \gg$. Le patient est ainsi conduit à consentir sans qu'il sache vraiment à quoi. La notion du consentement est au cœur et à l'origine des débats éthiques de la médecine moderne. Le problème du consentement, avant tout dans la recherche clinique, est lié aux procès des médecins nazis à Nuremberg ${ }^{9}$. La défense des avocats de ces médecins a consisté à dire qu'ils n'avaient pas agi en tant que Nazis, mais en tant que médecins, et que ce qui leur était reproché, d'avoir mené des expériences sur des condamnés à mort, était exactement ce que les Américains faisaient dans leurs prisons. Il est vrai, les Américains obtenaient, moyennant finances, un consentement de ces prisonniers pauvres, condamnés à mort, qui dès lors pouvaient cantiner, résider à l'infirmerie, etc. Aujourd'hui, au-delà de la recherche, c'est toute la pratique médicale qui devient une pédagogie de l'observance, et donc du consentement. Quelles sont les réponses possibles à ce discours de la médecine moderne scientifique et sociale, interroge Olivier Razac ${ }^{10}$ ? Dans cette perspective, la

6. J. Palazzolo et coll., L'observance médicamenteuse en psychiatrie, cité par A. Merlot, «De l'observance », op. cit., 2004.

7. Maud Mannoni ne voyait pas « de différence fondamentale entre l'éducation autoritaire et l'éducation "progressiste" ", dans cette dernière, il s'agit simplement de « persuader l'enfant qu'il est consentant». Cette remarque ne s'applique-t-elle pas au virage actuel de la position du médecin, tenu d'abjurer toute position «paternaliste » pour une relation «pédagogique » avec son patient ? Cf. M. Mannoni, Éducation impossible, Paris, Le Seuil, 1973.

8. A. Merlot, «De l'observance», op. cit.

9. G. J. Annas, M. A. Grodin, The Nazi Doctors and the Nurenberg Code. Human Rights in Human Experimentation, New York, Oxford University Press, 1992.

10. O. Razac, La grande santé, Paris, Climats, 2006. 
médicalisation est la modalité d'une «technologie à la fois individualisante et globalisante du pouvoir » qui caractérise la « société de contrôle » contemporaine. À partir des avancées de Foucault et Deleuze, et, au-delà de Nietzsche, ne peut-on aboutir qu'à une solution radicale, à « une forme générique de résistance à ce pouvoir » jusque-là «essentiellement disciplinaire » qui consiste en le « rejet du médecin, le refus de soin », et qui fut celle de nos campagnes, rétives longtemps à ce progrès qui mettait à mal les vieilles traditions, ou bien la nouvelle «société de contrôle» appelle-t-elle d'autres formes de résistance?

Ce noyau de la médecine moderne était souligné par Lacan dans sa conférence au Collège de médecine en 1966. La médecine se défausse sur le patient de sa responsabilité, et celui-ci, déjà en panne d'instances sociales qui prennent sur elles sa faute, sa culpabilité, la voit maintenant redoublée par le discours médical lui-même. Avec les campagnes actuelles, un déprimé ne pourra plus ignorer sa déprime, ni le protocole thérapeutique qui convient à son état. Il sait. Il saura. On notera au passage que ces maladies dites dépressives constituent l'une des principales causes d'arrêt de travail, les modifications historiques de celui-ci, son évolution, ses conditions ne sont, bien entendu, jamais interrogées dans ce cadre. On peut évoquer ici Hannah Arendt qui soulignait que

« nous nous sommes montrés assez ingénieux pour trouver les moyens de soulager la peine de vivre à tel point qu'il n'est plus utopique de songer à éliminer le travail du nombre des activités humaines. Car dès à présent, le mot travail est trop noble, trop ambitieux, pour désigner ce que nous faisons ou croyons faire dans le monde où nous sommes. Le dernier stade de la société de travail, la société d'employés, exige de ses membres un pur fonctionnement automatique, comme si la vie individuelle était réellement submergée par le processus global de la vie de l'espèce [...]. Ce qu'il y a de fâcheux dans les théories modernes du comportement, ce n'est pas qu'elles sont fausses, c'est qu'elles peuvent devenir vraies, c'est qu'elles sont en fait la meilleure mise en concept possible de certaines tendances évidentes de la société moderne ${ }^{11} »$.

11. H. Arendt, Condition de l'homme moderne, trad. G. Fradier, Paris, CalmannLévy, 1961. 
Elles peuvent devenir vraies, car nous n'avons jamais été modernes, pourrait-on dire avec Bruno Latour. Le grand partage cartésien qui a fondé notre modernité n'a jamais été absolu et ne s'est pas fait sans reste. Ce reste, c'est le fonds de la psychanalyse, son acte de naissance et encore sa chance. Le problème des protocoles actuels de l'EBM (evidence-based medecine), à savoir la validation statistique de protocoles thérapeutiques sur le modèle de l'oncologie, c'est qu'ils n'ont rien d'évident. Ainsi, l'évaluation des psychothérapies, effectuée par l'Inserm il y a quelques années, donne des résultats tout à fait différents de ceux pratiqués dans d'autres pays sur la même question.

Notre clinique quotidienne confirme bien que la dépression constitue une catégorie fluctuante, complexe et, surtout, transversale. Or, ce qui domine aujourd' hui est le retour à une nosographie « pinellienne » où le signe vaut pour le tableau clinique. Nous retrouvons ici le problème crucial de la disparition de la clinique dans la formation des médecins et des psychiatres, et prochainement dans celle des psychologues qu'on voudra continuer à dire abusivement cliniciens. Or, cette « vieille clinique » a partie plus ou moins liée depuis plusieurs décennies avec la psychanalyse qui lui a fourni une psychopathologie, l'a raisonnée, et, même, pour partie, réarticulée. Mais, si les «types cliniques » qu'elle dégage peuvent servir de repères, l'analyste ne peut en rester là. Quelque chose se réinvente constamment dans nos pratiques.

Le terme de dépression n'est pas fréquent chez Freud, mais quand il l'utilise, il parle littéralement de Genu $\beta$, c'est-à-dire jouissance. Longtemps, Lacan référera la notion de dépression à la position dépressive de Melanie Klein. Dans cette perspective, la possibilité dépressive est un moment de l'avènement du sujet, en ce sens peut-on parler de «bienfaits de la dépression », comme a pu le faire Pierre Fédida ${ }^{12}$. Mais la dépression ne suppose-t-elle pas un objet, dont la perte fait le deuil du déprimé, objet décisif puisque Lacan a pu dire qu'il n'a eu qu'à se laisser guider par " Deuil et mélancolie » pour inventer l'objet $a$, cet objet que Freud n'a pas su nommer. L'autre source de son invention étant l'objet transitionnel de Winnicott.

12. P. Fédida, Des bienfaits de la dépression. Éloge de la psychothérapie, Paris, Odile Jacob, 2001. 
La phase dépressive de Melanie Klein correspond au moment où la mère est réalisée comme totalité. Dès lors, l'enfant réparera cette totalité endommagée. La totalisation de la mère peut se comprendre aussi comme une perte d'objet, en tout cas, la perte d'une jouissance que j'imputerai dorénavant à l'Autre qui m'en dépossède. Rappelons que la mélancolie freudienne n'est ni une névrose, ni une psychose. Freud lui donnait une place limite entre les deux. Cette position limite devrait nous conduire à réinterroger nos repères, cette classification malgré tout psychiatrique dont nous usons, qui, si elle est incomparablement plus maniable et opérante que celle qui nous est aujourd'hui proposée, est néanmoins tout autant liée à son époque que celle-là. Nous devons donc les réinterroger, ne serait-ce qu'en partant de cette remarque que le mélancolique est cet objet, cet objet de la dépression, qui n'est rien d'autre que l'objet de Lacan. Autour de ce carrefour, autour de la place que tient l'objet, le statut de la dépression est à réarticuler versant psychose et versant névrose. Car la dépression, ou la dépressivité, est un marqueur de la mise en place de la structure, si l'on considère la phase dépressive. Elle est située par Lacan comme le lieu d'une première identification idéale. En retour, il y a une " prise de conscience de l'objet désiré en tant que tel », un autre « est en train de posséder le sein maternel» dont la totalisation me prive comme objet partiel. La notion de «partiel » devient problématique, puisque le sujet prend conscience de lui-même comme "privé ». Le sujet se sent privé parce qu'il est fondamentalement frustré, imaginairement frustré de ce qu'il voit, quand il retrouve cet objet, déduit de la plénitude qu'il suppose à cet autre qui enjouit.

Ce moment de la phase dépressive est fondamental, car il marque une perte de jouissance, perte de jouissance à l'origine du sujet, que l'on retrouve comme enjeu à la fin de la cure. C'est pourquoi Lacan pourra parler de deuil à la fin de l'analyse et d'une position plutôt « maniacodépressive ». Or, a-t-il pu dire, « cet objet que [Freud] n'a pas su nommer, l'objet privilégié, qu'il arrive qu'un être incarne pour nous ? » car «l'objet dont nous portons le deuil était à notre insu celui qui s'était fait le support de notre castration [...]. Nous sommes en deuil de quelqu'un dont nous pouvons dire "j'étais son manque" ». Cette dimension-là du deuil suppose une articulation du phallus. Mais, si l'identification 
à l'objet perdu fait partie de tout processus de deuil, dans la mélancolie, « l'ombre de l'objet tombe sur le moi ». Le sujet est identifié à l'objet, mais à l'objet comme déchet, à l'objet comme pure jouissance non phallique, quand l'objet auquel s'identifie le sujet dans le deuil est l'objet comme cause du désir. Du côté de l'objet pur, il n'y a pas de signifiant, cet objet n'est pas articulé à la «totalité » phallicisée de l'image. Lacan remarquera que les autoaccusations du mélancolique ne sont pas des autoaccusations narcissiques, «qu'il ne s'agit jamais de l'image spéculaire », car « il est entièrement dans ces autoaccusations dans le domaine du symbolique ». Ainsi, peut-on distinguer et conjoindre, dans cette perspective, ce que Lacan appelle " suicide de l'objet » pour la mélancolie de la «mort du sujet » dans la psychose. Le mélancolique devra passer « au travers de sa propre image », car le suicide est une tentative pour le sujet de rejoindre l'objet $a$, « il fait sauter le poinçon » de la formule du fantasme qui tient sur le versant névrotique.

Bien que se voulant athéorique dans la perspective du DSM-III, la notion de dépression renvoie à celle d'insuffisance, à des références implicites de la psychologie classique, celle de Janet. Lacan préfère la tristesse à la dépression, tristesse qui est une " faute morale, comme s'exprimait Dante, voire Spinoza : un péché, ce qui veut dire une lâcheté morale ${ }^{13} »$. Le mot lâcheté est à entendre aussi comme lâchage, et ce que le sujet lâche dans cette circonstance, c'est son désir. Il consent ainsi à être objet de jouissance, s'offrant à la jouissance, même si ça n'est pas une version psychotique. Il cède sur le « devoir de bien dire », le devoir «de s'y retrouver dans l'inconscient, dans la structure ", il lâche sur le symbolique pour se laisser aller à la jouissance, c'est la dépression du névrosé. Mais Lacan ajoute : «Et ce qui s'ensuit pour peu que cette lâcheté, d'être rejet de l'inconscient, aille à la psychose, c'est le retour dans le réel de ce qui est rejeté, du langage ; c'est l'excitation maniaque par quoi ce retour se fait mortel. » Que cette " lâcheté » puisse aller à la psychose sans rupture déjà là ouvre les questions que nous avons déjà évoquées sur le partage radical des types cliniques. N'y a-t-il pas là, avec cette référence à la tristesse, une nécessité de repenser l'articulation de cette

13. J. Lacan, Télévision, Paris, Le Seuil, 1973. 
typologie, héritée de la « vieille clinique » ? On soulignera l'interprétation du passage à l'acte mélancolique, conçu comme retour maniaque mortel où le sujet ne peut que rejoindre cet objet qui ne le leste plus. La tristesse est le péché car le sujet cède sur son désir et se retrouve face à la jouissance, ce que, littéralement, Freud dit de la dépression dans «Deuil et mélancolie » : il y a de la jouissance $($ Genu $\beta)$. « À l'opposé de la tristesse, il y a le gay sçavoir, lequel est une vertu », une vertu, c'est-à-dire pas sans courage. Le péché originel, bien sûr, " comme chacun sait » car il y a quelque chose d'originel dans la jouissance que le sujet rencontre dans le moment dépressif. L'analyse est orientée par le réel pour le contrer, le contrer par la vertu du bien dire. En fin de compte, « le sujet est heureux [...] il ne peut rien devoir qu'à l'heur, à la bonne fortune autrement dit», rencontres qui fondent leur propre répétition. En effet, «il n’y a de bonheur que du phallus ». Mais, pour autant, ce qui est méconnu par le sujet, c'est sa dépendance à la structure, son lien à l'Autre, qu'il situe comme lieu de la béatitude, mais dont il se sent «exilé ». Il se sent « exilé », ce qui est sa condition. Alors, péché ou « vraie touche du réel »? L'enjeu de ces moments dépressifs est renvoyé à la condition du sujet, ce qui peut se déchiffrer à la fin de la cure, et le problème de la tristesse rencontre celle de la fin del'analyse.

Ce lâchage est alimenté par les effets du discours contemporain qui, par le progrès des sciences, défait le sens, et c'est pourquoi, d'une certaine façon, le dépressif est celui qui est le mieux à même de nous apprendre quelque chose sur notre monde. Walter Benjamin, évoquant Kafka, pouvait écrire : «En effet, l'homme d'aujourd'hui vit dans son corps comme K. dans le village au pied du château ; il lui échappe, il lui est hostile. Il peut arriver que l'homme un matin se réveille et se trouve transformé en vermine. Le pays étranger - son pays étranger - s'est emparé de lui ${ }^{14} \ldots$ » Peut-on mieux dire l'objet de la dépression ? On peut aussi citer le livre d'Eduardo Carrasco Rahal ${ }^{15}$. Il est, me

14. W. Benjamin, «Franz Kafka. Pour le dixième anniversaire de sa mort » (1934), trad. M. de Gandillac, R. Rochlitz, P. Rusch, Euvres, t. II, Paris, Gallimard, 2000.

15. E. Carrasco Rahal, La place du fou. Autobiographie d'une psychose, Paris, Denoël, 2006. 
semble-t-il, le livre qui correspond à celui de Wolfson pour notre époque, et la dimension de l'humeur y prévaut. Un sujet décrit l'expérience folle qui a été la sienne, et l'on peut y lire, à partir d'un épisode aigu, exalté et délirant, une des plus extraordinaires descriptions des fictions modernes, dont il témoigne en s'en faisant le sacrifié. La monstration de la réduction généralisée de toute valeur à la valeur économique comme caractérisant notre monde est imparable, ce monde pour lequel Lacan proposait d'inventer une pratique sans valeur. Notre déprimé moderne, qui a le sentiment de ne rien valoir, coûte, selon un article du Monde, plus du double du non-déprimé : grand consommateur de consultations médicales, d'arrêts de travail, de médications diverses... La dépression est sans doute la marque que les gadgets ne gagnent pas encore, que "c'est pas ça », que nous ne sommes pas encore « vraiment animés par les gadgets ». Serons-nous à la hauteur de la question nouvelle que pose la dépression, formule éminemment contemporaine, mis en demeure que nous sommes de nous réinventer dans les termes de la crise contemporaine pour que notre pratique au un par un puisse encore se dire ? Lacan conseillait, en effet, à celui qui veut pratiquer la psychanalyse de renoncer, s'il «ne peut rejoindre à son horizon la subjectivité de son époque». 